\title{
Clause Size Reduction with all-UIP Learning
}

\author{
Nick Feng ${ }^{(\bowtie)}$ and Fahiem Bacchus ${ }^{(\bowtie)}$ \\ Department of Computer Science, University of Toronto, Toronto, Canada \\ \{fengnick,fbacchus\}@cs.toronto.edu
}

\begin{abstract}
Almost all CDCL SAT solvers use the 1-UIP clause learning scheme for learning new clauses from conflicts, and our current understanding of SAT solving provides good reasons for using that scheme. In particular, the 1-UIP scheme yields asserting clauses, and these asserting clauses have minimum LBD among all possible asserting clauses. As a result of these advantages, other clause learning schemes, like $i$-UIP and all-UIP, that were proposed in early work are not used in modern solvers. In this paper, we propose a new technique for exploiting the allUIP clause learning scheme. Our technique is to employ all-UIP learning under the constraint that the learnt clause's LBD does not increase (over the minimum established by the 1-UIP clause). Our method can learn clauses that are significantly smaller than the 1-UIP clause while preserving the minimum LBD. Unlike previous clause minimization methods, our technique is not limited to learning a sub-clause of the 1-UIP clause. We show empirically that our method can improve the performance of state of the art solvers.
\end{abstract}

\section{Introduction}

Clause learning is an essential technique in SAT solvers. There is good evidence to indicate that it is, in fact, the most important technique used in modern SAT solvers [6]. In early SAT research a number of different clause learning techniques were proposed $[5,19,20,25]$. However, following the revolutionary performance improvements achieved by the Chaff SAT solver, the field has converged on using the 1-UIP (first Unique Implication Point) scheme [25] employed in Chaff [13] (as well as other techniques pioneered in the Chaff solver). ${ }^{1}$ Since then almost all SAT solvers have employed the 1-UIP clause learning scheme, along with clause minimization [21], as their primary method for learning new clauses.

However, other clause learning schemes can be used in SAT solvers without changes to the main data structures. Furthermore, advances in our understanding allow us to better understand the potential advantages and disadvantages of these alternate schemes. In this paper we reexamine these previously proposed schemes with a focus on the schemes described in [25]. Improved understanding

\footnotetext{
1 The idea of UIP clauses was first mentioned in [19], and 1-UIP clauses along with other UIP clauses were learnt and used in the earlier GRASP SAT solver.

(C) Springer Nature Switzerland AG 2020

L. Pulina and M. Seidl (Eds.): SAT 2020, LNCS 12178, pp. 28-45, 2020.

https://doi.org/10.1007/978-3-030-51825-7_3
} 
of SAT solvers, obtained from the last decade of research, allows us to see that in their original form these alternative clause learning schemes suffer significant disadvantages over 1-UIP clause learning.

One of the previously proposed schemes was the all-UIP scheme [25]. In this paper we propose a new way to exploit the main ideas of this scheme that avoids its main disadvantage which is that it can learn clauses with higher LBD scores. In particular, we propose to use a all-UIP like clause learning scheme to generate smaller learnt clauses which retain the good properties of standard 1-UIP clauses. Our method is related to, but not the same as, various clause minimization methods that try to remove redundant literals from the 1-UIP clause yielding a clause that is a subset of the 1-UIP clause, e.g., [10,21,24]. Our method is orthogonal to clause minimization. In particular, our approach can learn a clause that is not a subset of the 1-UIP clause but which still serves all of the same purposes as the 1-UIP clause. Clause minimization techniques can be applied on top of our method to remove redundant literals.

We present various versions of our method and show that these variants are often capable of learning shorter clauses than the 1-UIP scheme, and that this can lead to useful performance gains in state of the art SAT solvers.

\section{Clause Learning Framework}

We first provide some background and a framework for understanding clause learning as typically used in CDCL SAT solvers. A propositional formula $F$ expressed in Conjunctive Normal Form (CNF) contains a set of variables $V$. A literal is a variable $v \in V$ or its negation $\neg v$. For a literal $\ell$ we let $\operatorname{var}(\ell)$ denote its underlying variable. A CNF consists of a conjunction of clauses, each of which is a disjunction of literals. We often view a clause as being a set of literals and employ set notation, e.g., $\ell \in C$ and $C^{\prime} \subset C$.

Two clauses $C_{1}$ and $C_{2}$ can be resolved when they contain conflicting literals $\ell \in C_{1}$ and $\neg \ell \in C_{2}$. Their resolvent $C_{1} \bowtie C_{2}$ is the new clause $\left(C_{1} \cup C_{2}\right)-\{\ell, \neg \ell\}$. The resolvent will be a tautology (i.e., a clause containing a literal $x$ and its negation $\neg x)$ if $C_{1}$ and $C_{2}$ contain more than one pair of conflicting literals.

We assume the reader is familiar with the operations of CDCL SAT solvers, and the main data structures used in such solvers. A good source for this background is [18].

The Trail. CDCL SAT solvers maintain a trail, $\mathcal{T}$, which is a non-contradictory, non-redundant sequence of literals that have been assigned TRUE by the solver; i.e. $\ell \in \mathcal{T} \rightarrow \neg \ell \notin \mathcal{T}$, and $\mathcal{T}$ contains no duplicates. Newly assigned literals are added to the end of the trail, and on backtrack literals are removed from the end of the trail and unassigned. If literal $\ell$ is on the trail let $\iota(\ell)$ denote its index on the trail, i.e, $\mathcal{T}[\iota(\ell)]=\ell$. For convenience, we also let $\iota(\ell)=\iota(\neg \ell)=\iota(\operatorname{var}(\ell))$ even though neither $\neg \ell$ nor $\operatorname{var}(\ell)$ are actually on $\mathcal{T}$. If $x$ and $y$ are both on the trail and $\iota(x)<\iota(y)$ we say that $x$ appears before $y$ on the trail.

Two types of true literals appear on the trail: decision literals that have been assumed to be true by the solver, and unit propagated literals that are forced to 
be true because they are the sole remaining unfalsified literal of a clause. Each literal $\ell \in \mathcal{T}$ has a decision level $\operatorname{dec} L v l(\ell)$. Let $k$ be the number of decision literals appearing before $\ell$ on the trail. When $\ell$ is a unit propagated literal $\operatorname{dec} \operatorname{Lvl}(\ell)=k$, and when $\ell$ is a decision literal $\operatorname{dec} \operatorname{Lvl}(\ell)=k+1$. For example, $\operatorname{dec} \operatorname{Lvl}(d)=1$ for the first decision literal $d \in \mathcal{T}$, and $\operatorname{dec} \operatorname{Lvl}(\ell)=0$ for all literals $\ell$ appearing before $d$ on the trail. The set of literals on $\mathcal{T}$ that have the same decision level forms a contiguous subsequence of $\mathcal{T}$ that starts with a decision literal $d_{i}$ and ends just before the next decision literal $d_{i+1}$. If $\operatorname{dec} \operatorname{Lvl}\left(d_{i}\right)=i$ we call this subsequence of $\mathcal{T}$ the $i$-th decision level.

Each literal $\ell \in \mathcal{T}$ also has a clausal reason reason $(\ell)$. If $\ell$ is a unit propagated literal, reason $(\ell)$ is a clause of the formula such that $\ell \in$ reason $(\ell)$ and $\forall x \in$ reason $(\ell) . x \neq \ell \rightarrow(\neg x \in \mathcal{T} \wedge \iota(\neg x)<\iota(\ell))$. That is, reason $(\ell)$ is a clause that has become unit implying $\ell$ due to the literals on the trail above $\ell$. If $\ell$ is a decision literal then reason $(\ell)=\varnothing$.

In most SAT solvers, clause learning is initiated as soon as a clause is falsified by $\mathcal{T}$. In this paper we will be concerned with the subsequent clause learning process which uses $\mathcal{T}$ to derive a new clause. We will try to make as few assumptions about how $\mathcal{T}$ is managed by the SAT solver as possible. One assumption we will make is that $\mathcal{T}$ remains intact during clause learning and is only changed after the new clause is learnt (by backtracking).

Say that $\mathcal{T}$ falsifies a clause $C_{I}$, and that the last decision literal $d_{k}$ in $\mathcal{T}$ has decision level $k$. Consider $\mathcal{T}_{k-1}$ the prefix of $\mathcal{T}$ above the last decision level, i.e., the sequence of literals $\mathcal{T}[0]-\mathcal{T}\left[\iota\left(d_{k}\right)-1\right]$. We will assume that $\mathcal{T}_{k-1}$ is unit propagation complete, although the full trail $\mathcal{T}$ might not be. This means that (a) no clause was falsified by $\mathcal{T}_{k-1}$. And (b) if $C_{u}$ is a clause containing the literal $x$ and all literals in $C_{u}$ except for $x$ are falsified by $\mathcal{T}_{k-1}$, then $x \in \mathcal{T}_{k-1}$ and $\operatorname{decLvl}(x) \leq \max \left\{\operatorname{decLvl}(y) \mid y \in C_{u} \wedge y \neq x\right\}$. This means that if $x$ appears in a clause made unit it must have been added to the trail, and added at or before decision level the clause became unit. Note that more than one clause might be made unit by $\mathcal{T}$ forcing $x$, or $x$ might be set as a decision before being forced. This condition ensures that $x$ appears in $\mathcal{T}$ at or before the first decision level it is forced by any clause.

Any clause falsified by $\mathcal{T}$ is called a conflict. When a conflict is found, the final level of the trail, $k$, need not be unit propagation complete as the solver typically stops propagation as soon as it finds a conflict. This means that (a) other clauses might be falsified by $\mathcal{T}$ besides the conflict found, and (b) other literals might be unit implied by $\mathcal{T}$ but not added to $\mathcal{T}$.

Definition 1 (Trail Resolvent). A trail resolvent is a clause arising from resolving a conflict against the reason clause of some literal $\ell \in \mathcal{T}$. Every trail resolvent is also a conflict.

The following things can be noted about trail resolvents: (1) trail resolvents are never tautological, as the polarity of all literals in reason $(\ell)$ other than $\ell$ must agree with the polarity of all literals in the conflict (they are all falsified by $\mathcal{T})$; $(2)$ one polarity of the variable $\operatorname{var}(\ell)$ resolved on must be a unit propagated literal whose negation appears in the conflict; and (3) any variable in the conflict 
that is unit propagated in $\mathcal{T}$ can be resolved upon (the variable must appear in different polarities in the conflict and in $\mathcal{T}$ ).

Definition 2 (Trail Resolution). A trail resolution is a sequence of trail resolvents applied to an initial conflict $C_{I}$ yielding a new conflict $C_{L}$. A trail resolution is ordered if the sequence of variables $v_{1}, \ldots, v_{m}$ resolved have strictly decreasing trail indices: $\iota\left(v_{i+1}\right)<\iota\left(v_{i}\right)(1 \leq i<m)$. (Note that this implies that no variable is resolved on more than once).

Ordered trail resolutions resolve unit propagated literals from the end of the trail to the beginning. W.l.o.g we can require that all trail resolutions be ordered.

Observation 1. If the unordered trail resolution $U$ yields the conflict clause $C_{L}$ from an initial conflict $C_{I}$, then there exists an ordered trail resolution $O$ that yields a conflict clause $C_{L}^{\prime}$ such that $C_{L}^{\prime} \subseteq C_{L}$.

Proof. Let $U$ be the sequence of clauses $C_{I}=C_{0}, C_{1}, \ldots, C_{m}=C_{L}$ obtained by resolving on the sequence of variables $v_{1}, \ldots, v_{m}$ whose corresponding literals on $\mathcal{T}$ are $l_{1}, \ldots, l_{m}$. Reordering these resolution steps so that the variables are resolved in order of decreasing trail index and removing duplicates yields an ordered trail resolution $O$ with the desired properties. Since no reason clause contains literals with higher trail indices, $O$ must be a valid trail resolution if $U$ was, and furthermore $O$ yields the clause $C_{L}^{\prime}=\bigcup_{i=1}^{m}$ reason $\left(l_{i}\right)-\left\{l_{1}, \neg l_{1}, \ldots, l_{m}, \neg l_{m}\right\}$. Since $U$ resolves on the same variables (in a different order) using the same reason clauses we must have $C_{L}^{\prime} \subseteq C_{L}$. It can, however, be the case that $C_{L}^{\prime}$ is proper subset of $C_{L}$ : if $l_{i}$ is resolved away it might be reintroduced when resolving on $l_{i+1}$ if $\iota\left(l_{i+1}\right)>\iota\left(l_{i}\right)$.

The relevance of trail resolutions is that all proposed clause learning schemes we are aware of use trail resolutions to produce learnt clauses. Furthermore, the commonly used technique for clause minimization [21] is also equivalent to a trail resolution that yields the minimized clause from the un-minimized clause. Interestingly, it is standard in SAT solver implementations to perform resolution going backwards along the trail. That is, these implementations are typically using ordered trail resolutions. Observation 1 shows that this is correct.

Ordered trail resolutions are a special case of trivial resolutions [2]. Trail resolutions are specific to the trail data structure typically used in SAT solvers. If $\mathcal{T}$ falsifies a clause at its last decision level, then its associated implication graph [20] contains a conflict node. Cuts in the implication graph that separate the conflict from the rest of the graph correspond to conflict clauses [2]. It is not difficult to see that the proof Proposition 4 of [2] applies also to trail resolutions. This means that any conflict clause in the trail's implication graph can be derived using a trail resolution.

\subsection{Some Alternate Clause Learning Schemes}

A number of different clause learning schemes for generating a new learnt clause from the initial conflict have been presented in prior work, e.g., [5, 19, 20,25]. 


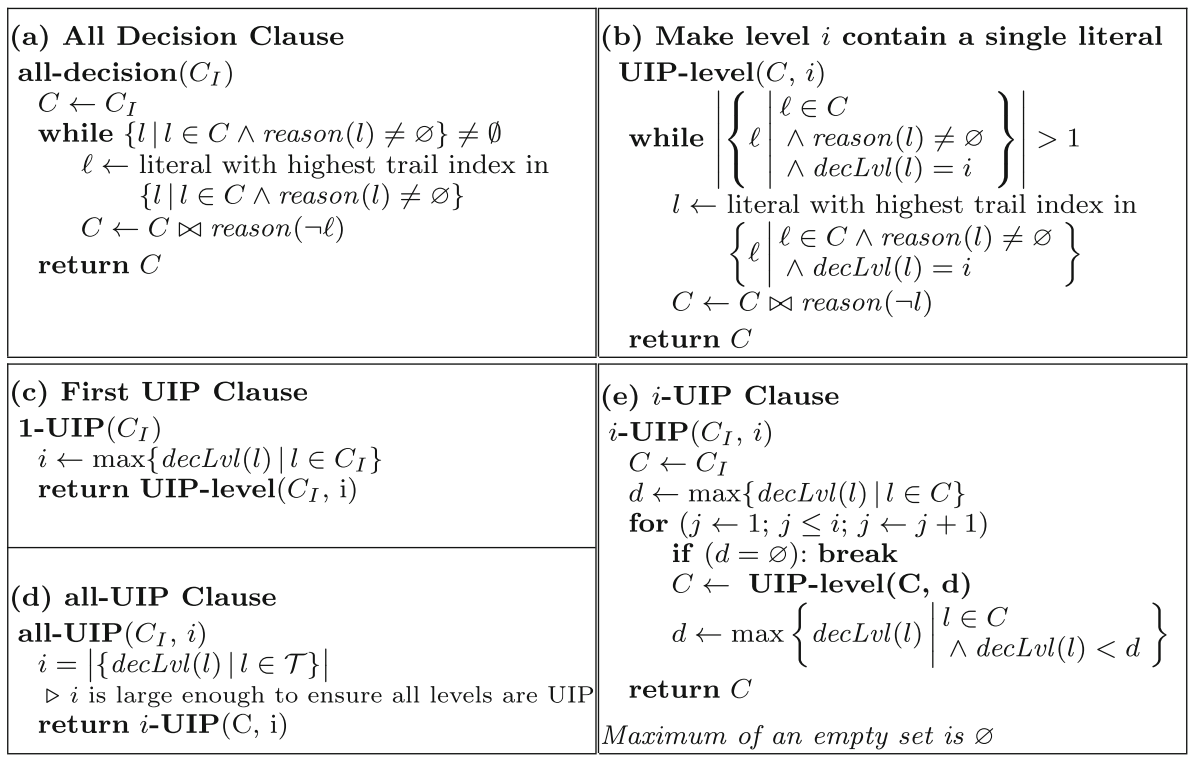

Fig. 1. Some different clause learning schemes. All use the current trail $\mathcal{T}$ and take as input an initial clause $C_{I}$ falsified by $\mathcal{T}$ at its deepest level.

Figure 1 gives a specification of some of these methods: (a) the all-decision scheme which resolves away all implied literals leaving a learnt clause over only decision literals; (c) the 1-UIP scheme which resolves away literals from the deepest decision level leaving a learnt clause with a single literal at the deepest level; (d) the all-UIP scheme which resolves away literals from each decision level leaving a learnt clause with a single literal at each decision level; and (e) the $i$-UIP scheme which resolves away literals from the $i$ deepest decision levels leaving a learnt clause with a single literal at its $i$ deepest decision levels. It should be noted that when resolving away literals at decision level $i$, new literals at decision levels less than $i$ might be introduced into the clause. Hence, it is important in the $i$-UIP and all-UIP schemes to use ordered trail resolutions.

Both the all-decision and all-UIP schemes yield a clause with only one literal at each decision level, and the all-UIP clause will be no larger that the alldecision clause. Furthermore, it is known [20] that once we reduce the number of literals at a decision level $d$ to one, we could continue performing resolutions and later achieve a different single literal at the level $d$. In particular, a decision level might contain more than one unique implication point, and in some contexts the term all-UIP is used to refer to all the unique implication points that exist in a particular decision level [17] rather than the all-UIP clause learning scheme as is used here. The algorithms given in Fig. 1 stop at the first UIP of a level, except for the all-decision schemes with stops at the last UIP of each level. 


\subsection{Asserting Clauses and LBD-Reasons to Prefer 1-UIP Clauses}

An asserting clause [15] is a conflict clause $C_{L}$ that has exactly one literal $\ell$ at its deepest level, i.e., $\forall x \in C_{L} \cdot \operatorname{dec} L v l(x) \leq \operatorname{dec} L v l(\ell) \wedge(\operatorname{dec} L v l(x)=\operatorname{dec} L v l(\ell) \rightarrow$ $x=\ell$ ). All of the clause learning schemes in Fig. 1 produced learnt clauses that are asserting.

The main advantage of asserting clauses is that they are 1-Empowering [15], i.e., they allow unit propagation to derive a new forced literal. Hence, asserting clauses can be used to guide backtracking - the solver can backtrack from the current deepest level to the point the learnt clause first becomes unit, and then use the learnt clause to add a new unit implicant to the trail. Since all but the deepest level was unit propagation complete, this means that the asserting clause must be a brand new clause; otherwise that unit implication would already have been made. On the other hand, if the learnt clause $C_{L}$ is not asserting then it could be that it is a duplicate of another clause already in the formula.

Example 1. Suppose that $a$ is a unit propagated literal and $d$ is a decision literal with $\operatorname{dec} L v l(d)>\operatorname{dec} L v l(a)$. Let the sequence of clauses watched by $\neg d$ be $(\neg d, x$, $\neg a),(\neg d, y, \neg x, \neg a),(\neg d, \neg y, \neg x, \neg a),(\neg d, \neg x, \neg a)$. When $d$ is unit propagated the clauses on $\neg d$ 's watch list will be checked in this order.

Hence, unit propagation of $d$ will extend the trail by first adding the unit propagated literal $x$ (with reason $(x)=(x, \neg d, \neg a)$ ) and then the unit propagated literal $y$ (with reason $(y)=(y, \neg x, \neg a, \neg d))$. Now the third clause on $\neg d$ 's watch list, $(\neg d, \neg y, \neg x, \neg a)$ is detected to be a conflict.

Clause learning can now be initiated from conflict $C_{I}=(\neg d, \neg y, \neg x, \neg a)$. This clause has 3 literals at level $\operatorname{dec} \operatorname{Lvl}(d)=10$. If we stop clause learning before reaching an asserting clause, then it is possible to simply resolve $C_{I}$ with reason $(y)$ to obtain the learnt clause $C_{L}=(\neg d, \neg x, \neg a)$. However, this nonasserting learnt clause is a duplicate of the fourth clause on $\neg d$ 's watch list which is already in the formula. ${ }^{2}$ This issue can arise whenever $C_{L}$ contains two or more literals at the deepest level (i.e., whenever $C_{L}$ is not asserting). In such cases $C_{L}$ might be a clause already in the formula with its two watches not yet fully unit propagated (and thus $C_{L}$ is not detected by the SAT solver to be a conflict) since propagation is typically stopped as soon as a conflict is detected.

The LBD of the learnt clause $C_{L}$ is the number of different decision levels in it: $\operatorname{LBD}\left(C_{L}\right)=\left|\left\{\operatorname{dec} L v l(l) \mid l \in C_{L}\right\}\right|$ [1]. Empirically LBD is a successful predictor of clause usefulness: clauses with lower LBD tend to be more useful. As noted in [1], from the initial falsified clause $C_{I}$ the 1-UIP scheme will produce a clause $C_{L}$ whose LBD is minimum among all asserting clauses that can be learnt from $C_{I}$. If $C^{\prime}$ is a trail resolvent of $C$ and a reason clause reason $(l)$, then $\operatorname{LBD}\left(C^{\prime}\right) \geq \operatorname{LBD}(C)$ since reason $(l)$ must contain at least one other literal

\footnotetext{
${ }^{2}$ In this example, the fourth clause on $\neg d$ 's watch list subsumes the third clause. But it is not difficult to construct more elaborate examples where there are no subsumed clauses and we still obtain learnt clauses that are duplicates of clauses already in the formula.
} 
with the same decision level as $l$ and might contain literals with decision levels not in $C$. That is, the each trail resolution step might increase the LBD of the learnt clause and can never decrease the LBD. Hence, the 1-UIP scheme yields an asserting clause with minimum LBD as it performs the minimum number of trail resolutions required to generate an asserting clause.

The other schemes must perform more trail resolutions. In fact, all of these schemes (all-decision, all-UIP, i-UIP) use trail resolutions in which the 1-UIP clause appears. That is, they all must first generate the 1-UIP clause and then continue with further trail resolution steps. These extra resolution steps can introduce many addition decision levels into the final clause. Hence, these schemes learn clauses with LBD at least as large as the 1-UIP clauses.

Putting these two observations together we see that the 1-UIP scheme produces asserting clauses with lowest possible LBD. This is a compelling reasons for using this scheme. Hence, it is not surprising that modern SAT solvers almost exclusively use 1 -UIP clause learning. ${ }^{3}$

\section{Using all-UIP Clause Learning}

Although learning clauses with low LBD has been shown empirically to be more important in SAT solving than learning short clauses [1], clause size is still important. Smaller clauses consume less memory and help to decrease the size of future learnt clauses. They are also semantically stronger than longer clauses.

The all-UIP scheme will tend to produce small clauses since the clauses contain at most one literal per decision level. However, the all-UIP clause can have much higher LBD. Since LBD is more important than size, our approach is to use all-UIP learning when, and only when, it succeeds in reducing the size of the clause without increasing its LBD. The all-UIP scheme first computes the 1-UIP clause when it reduces the deepest level to a single UIP literal. It then proceeds to reduce the shallower levels (see all-UIP's for loop in Fig. 1). So our approach will start with the 1-UIP clause and then try to apply all-UIP learning to reduce other levels to single literals. As noted above, clause minimization is orthogonal to our approach, so we also first apply standard clause minimization [21] to the 1-UIP clause. That is, our algorithm stable-alluip (Algorithm 1), starts with the clause that most SAT solvers learn from a conflict, a minimized 1-UIP clause.

Algorithm 1 tries to compute a clause shorter than the inputted 1-UIP clause $C_{1}$. If a clause shorter than $C_{1}$ cannot be computed the routine returns $C_{1}$ unchanged. Line 2 uses the parameter $t_{\text {gap }}$ to predict if Algorithm 1 will be successful in producing a shorter clause. This predication is described below. If the prediction is negative $C_{1}$ is immediately returned and Algorithm 1 is not attempted. Otherwise, a copy of $C_{1}$ is made in $C_{i}$ and $n_{\text {tries }}$, which counts the number of times Algorithm 1 is attempted, is incremented.

\footnotetext{
${ }^{3}$ Knuth in his sat13 CDCL solver [7] uses an all-decision clause when the 1-UIP clause is too large. In this context an all-UIP clause could also be used as it would be no larger than the all decision clause.
} 


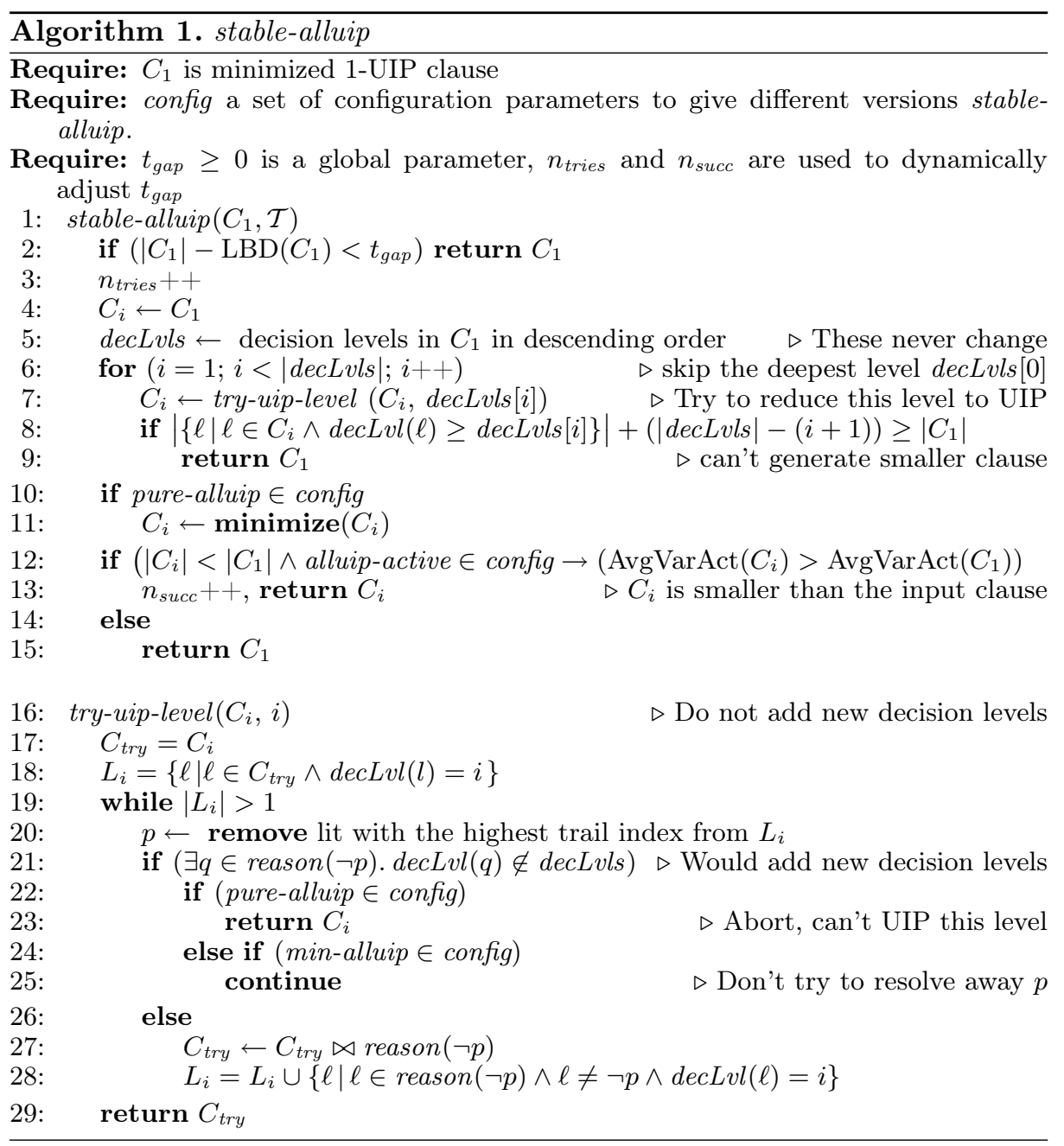

Then the decision levels of $C_{1}$ are computed and stored in decLvls in order from largest to lowest. The for loop of lines 6-9 is then executed for each decision level decLvls[i]. In the loop the subroutine try-uip-level tries to reduce the set of literals at decLvls[i] down to a single UIP literal using a sequence of trail resolutions. Since $C_{1}$ is a 1 -UIP clause decLvls[0] (the deepest level) already contains only one literal, so we can start at $i=1$.

After the call to try-uip-level a check (line 8) is made to see if we can abort further processing. At this point the algorithm has finished processing levels decLvls $[0]-d e c L v l s[i]$ so the literals at those levels will not change. Furthermore, we know that the best that can be done from this point on is to reduce the remaining $\mid$ decLvls $\mid-(i+1)$ levels down to a single literal each. Hence, adding these two numbers gives a lower bound on the size of the final computed clause. 
If that lower bound is as large as the size of the initial 1-UIP clause we can terminate and return the initial 1-UIP clause.

After processing all decision levels, if try-uip-level is using the pure-alluip configuration, additional reduction in the clause size might be achieved by an another round of clause minimization (line 11). Finally, if the newly computed clause $C_{i}$ is smaller that the input clause $C_{1}$ it is returned. Otherwise the original clause $C_{1}$ is returned. Additionally, if the configuration alluip-active, described in Sect.3.1, is being used, then we also require that the average activity level of the new clause $C_{i}$ be larger than $C_{1}$ before we can return the new clause $C_{i}$.

try-uip-level $\left(C_{i}, i\right)$ attempts to resolve away the literals at decision level $i$ in the clause $C_{i}$, i.e., those in the set $L_{i}$ (line 18), in order of decreasing trail index, until only one literal at level $i$ remains. If the resolution step will not introduce any new decision levels (line 26), it is performed updating $C_{t r y}$. In addition, all new literals added to $C_{\text {try }}$ at level $i$ are added to $L_{i}$.

On the other hand, if the resolution step would introduce new decision levels (line 21) then there are two options. The first option we call pure-alluip. With pure-alluip we abort our attempt to UIP this level and return the clause with level $i$ unchanged. In the second option, called min-alluip, we continue without performing the resolution, keeping the current literal $p$ in $C_{t r y}$. min-alluip then continues to try to resolve away the other literals in $L_{i}$ (note that $p$ is no longer in $L_{i}$ ) until $L_{i}$ is reduced to a single literal. Hence, min-alluip can leave multiple literals at level $i$-all of those with reasons containing new levels along with one other. ${ }^{4}$ Observe that the number of literals at level $i$ can not be increased after processing it with pure-alluip. min-alluip can, however, potentially increase the number of literals at level $i$. In resolving away a literal $l$ at level $i$, more literals might be introduced into level $i$, and some of these might not be removable by min-alluip if their reasons contain new levels. However, both pure-alluip and min-alluip can increase the number of literals at levels less that $i$ as new literals can be introduced into those levels when the literals at level $i$ are resolved away. These added literals at the lower levels might not be removable from the clause, and thus both methods can yield a longer clause than the input 1-UIP clause.

After trying to UIP each level the clause $C_{i}$ is obtained. If we were using pure-alluip we can once again apply recursive clause minimization (line 11) [21], but this would be useless when using min-alluip as all but one literal of each level introduces a new level and thus cannot be recursively removed. ${ }^{5}$

$t_{\text {gap }}$ : stable-alluip can produce significantly smaller clauses. However, when it does not yield a smaller clause, the cost of the additional resolution steps can hurt the solver's performance. Since resolution cannot reduce a clause's LBD, the maximum size reduction obtainable from stable-alluip is the difference between the 1-UIP clause's size and its LBD: $\operatorname{gap}\left(C_{1}\right)=\left|C_{1}\right|-\operatorname{LBD}\left(C_{1}\right)$. When $\operatorname{gap}\left(C_{1}\right)$

\footnotetext{
${ }^{4}$ Since the sole remaining literal $u \in L_{i}$ is at a lower trail index than all of the other literals there is no point in trying to resolve away $u$ - either it will be the decision literal for level $i$ having no reason, or its reason will contain at least one other literal at level $i$.

${ }^{5}$ Other more powerful minimization techniques could still be applied.
} 
is small, applying stable-alluip is unlikely to be cost effective. Our approach is to dynamically set a threshold on $\operatorname{gap}\left(C_{1}\right), t_{\text {gap }}$, such that when $\operatorname{gap}\left(C_{1}\right)<t_{\text {gap }}$ we do not attempt to reduce the clause (line 2). Initially, $t_{\text {gap }}=0$, and we count the number of times stable-alluip is attempted $\left(n_{\text {tries }}\right)$ and the number of times it successfully yields a shorter clause $\left(n_{\text {succ }}\right)$ (line 3 and 13 ). On every restart if the success rate since the last restart is greater than $80 \%$ (less than $80 \%$ ), we decrease (increase) $t_{\text {gap }}$ by one not allowing it to become negative.

Example 2. Consider the trail $\mathcal{T}=\ldots, \ell_{1}, a_{2}, b_{2}, c_{2}, d_{2}, \ldots, \ldots, e_{5}, f_{5}, g_{5}, h_{6}$, $i_{6}, j_{6}, k_{6}, \ldots, m_{10}, \ldots$ where the subscript indicates the decision level of each literal and the literals are in order of increasing trail index.

\begin{tabular}{|l|l|l|}
\hline$C_{a}=\varnothing$ & $C_{b}=\left(b_{2}, \neg \ell_{3}, \neg a_{2}\right)$ & $C_{c}=\left(c_{2}, \neg a_{2}, \neg b_{2}\right)$ \\
\hline$C_{d}=\left(d_{2}, \neg b_{2}, \neg c_{2}\right)$ & $C_{\ell}=\varnothing$ & $C_{e}=\varnothing$ \\
\hline$C_{f}=\left(f_{5}, \neg e_{5}, \neg \ell_{1}\right)$ & $C_{g}=\left(g_{5}, \neg a_{2}, \neg f_{5}\right)$ & $C_{h}=\varnothing$ \\
\hline$C_{i}=\left(i_{6}, \neg e_{5}, \neg h_{6}\right)$ & $C_{j}=\left(j_{6}, \neg f_{5}, \neg i_{6}\right)$ & $C_{k}=\left(k_{6}, \neg f_{5}, \neg j_{6}\right)$ \\
\hline
\end{tabular}

Let the clauses $C_{x}$, show above, denote the reason clause for literal $x_{i}$. Suppose 1-UIP learning yields the clause $C_{1}=\left(\neg m_{10}, \neg k_{6}, \neg j_{6}, \neg i_{6}, \neg h_{6}, \neg g_{5}, \neg d_{2}, \neg c_{2}\right)$ where $\neg m_{10}$ is the UIP from the conflicting level. stable-alluip first tries to find the UIP for level 6 by resolving $C_{1}$ with $C_{k}, C_{j}$ and then $C_{i}$ producing the clause $C^{*}=\left(\neg m_{10}, \neg h_{6}, \neg g_{5}, \neg f_{5}, \neg e_{5}, \neg d_{2}, \neg c_{2}\right)$ where $\neg h_{6}$ is the UIP for level 6 .

stable-alluip then attempts to find the UIP for level 5 by resolving $C^{*}$ with $C_{g}$ and then $C_{f}$. However, resolving with $C_{f}$ would introduce $\ell_{1}$ and a new decision level into $C^{*}$. pure-alluip thus leaves level 5 unchanged. min-alluip, on the other hand, skips the resolution with $C_{f}$ leaving $f_{5}$ in $C^{*}$. Besides $f_{5}$ only one other literal at level 5 remains in the clause, $e_{5}$, so min-alluip does not do any further resolutions at this level. Hence, pure-alluip yields $C^{*}$ unchanged, while min-alluip yields $C_{\text {min }}^{*}=\left(\neg m_{10}, \neg h_{6}, \neg f_{5}, \neg e_{5}, \neg d_{2}, \neg c_{2}, \neg a_{2}\right)$.

Finally, stable-alluip processes level 2. Resolving away $d_{2}$ and then $c_{2}$ will lead to an attempt to resolve away $b_{2}$. But again this would introduce a new decision level with the literal $\ell_{1}$. So pure-alluip will leave level 2 unchanged and min-alluip will leave $b_{2}$ unresolved. The final clauses produced by pure-alluip would be $\left(\neg m_{10}, \neg h_{6}, \neg f_{5}, \neg e_{5}, \neg d_{2}, \neg c_{2}, \neg a_{2}\right)$, a reduction of 1 over the 1-UIP clause, and by min-alluip would be ( $\left.\neg m_{10}, \neg h_{6}, \neg f_{5}, \neg e_{5}, \neg b_{2}, \neg a_{2}\right)$, a reduction of 2 over the 1-UIP clause.

\subsection{Variants of stable-alluip}

We also developed and experimented with a few variants of the stable-alluip algorithm which we describe below.

alluip-active: Clauses with Active Variables. stable-alluip learning might introduce literals with low variable activity into $C_{i}$. Low activity variables are variables that have had low recent participation in clause learning. Hence, clauses with variables of low activity might not be as currently useful to the solver. Our variant alluip-active (line 12) in Algorithm 1) computes the average variable activity of the newly produced all-UIP clause $C_{i}$ and the original 1-UIP clause 
$C_{1}$. The new clause $C_{i}$ will be returned only if it is both smaller and has higher average variable activity than the original 1-UIP clause. There are, of course, generalizations of this approach where one has a weighted trade-off between these factors that allows preferring the new clause when it has large gains in one metric even though it has small losses in the other. We did not, however, experiment with such generalizations.

Adjust Variable Activity. An alternative to filtering clauses with low average variable (alluip-active) is to alter the way variable activities are updated to account for our new clause learning method. The popular branching heuristics VSIDS [13] and LBR [8] bump the variable activity for all literals appearing in the learnt clause $C_{L}$ and all literals resolved away during the conflict analysis that yielded $C_{L}$ from the initially detected conflict $C_{I}$ (all literals on the conflict side).

We did not apply this approach to the stable-alluip clause, as we did not want to bump the activity of the literals above the deepest decision level that stable-alluip resolves away. Intuitively, these literals did not directly contribute to generating the conflict. Instead, we tried two modifications to the variable activity bumping schemes.

Let $C_{1}$ be the 1-UIP learnt clause and $C_{i}$ be the stable-alluip learnt clause. First, we kept all of the variable activity bumps normally done by 1-UIP learning. ${ }^{6}$ Then, when the stable-alluip scheme was successful, i.e., $C_{i}$ was to be used as the new learnt clause, we perform further updates to the variable activities. In the alluip-inclusive approach all variables variables appearing in $C_{i}$ that are not in $C_{1}$ have their activities bumped. Intuitively, since the clause $C_{i}$ is being added to the clause database we want to increase the activity of all of its variables. On the other hand, in the alluip-exclusive approach in addition to bumping the activity of the new variables in $C_{i}$ we also remove the activity bumps of those variables in $C_{1}$ that are no longer in $C_{i}$.

In sum, the two modified variable activity update schemes we experimented with were (1) alluip-inclusive $\equiv \forall l \in C_{i}-C_{1}$.bumpActivity $(l)$ and (2) alluip-exclusive $\equiv \forall l \in C_{i}-C_{1}$. bumpActivity $(l) \wedge\left(\forall l \in C_{1}-\right.$ $C_{i}$. unbumpActivity $\left.(l)\right)$.

Chronological Backtracking. We tested our new clause learning schemes on solvers that utilized Chronological Backtracking $[12,14]$. When chronological backtracking is used, the literals on the trail might no longer be sorted by decision level. So resolving literals in the conflict by highest trail index first no longer works. However, we can define a new ordering on the literals to replace the trail index ordering. Let $l_{1}$ and $l_{2}$ be two literals on the trail $\mathcal{T}$. We say that $l_{1}>_{\text {chron }} l_{2}$ if $\operatorname{decLvl}\left(l_{1}\right)>\operatorname{dec} \operatorname{Lvl}\left(l_{2}\right) \vee\left(\operatorname{decLvl}\left(l_{1}\right)=\operatorname{decLvl}\left(l_{2}\right) \wedge \iota\left(l_{1}\right)>\iota\left(l_{2}\right)\right)$. That is, literals with higher decision level come first, and if that is equal then the literal with higher trail index comes first.

${ }^{6}$ So extra techniques used by the underlying solver, like reason side rate and locality [8], were kept intact. 
Exploiting the analysis of [12], it can be observed that all clause learning schemes continue to work as long as literals are resolved away from the initial conflict in decreasing $>_{\text {chron }}$ order. In our implementation we used a heap (priority queue) to achieve this ordering of the literal resolutions in order to add our new schemes to those solvers using chronological backtracking.

\section{Implementation and Experiments}

We implemented stable-alluip learning schemes on MapleCOMSPS-LRB [9], the winner of SAT Race 2016 application track. We then evaluated these schemes and compare against the 1-UIP baseline on the full set of benchmarks from SAT RACE 2019 main track which contains 400 instances. We ran our experiments on $2.70 \mathrm{GHz}$ XeonE5-2680 CPUs, allowing 5000 seconds per instance and a maximum of 12 GB memory.

\begin{tabular}{|l|l|l|l|}
\hline Solver & \# solved (SAT, UNSAT) & PAR-2 & avg. clause Size \\
\hline 1-UIP & $221(132,89)$ & 5018.89 & 62.6 \\
\hline pure-alluip & $\mathbf{2 2 8}(135, \mathbf{9 3})+\mathbf{7}$ & 4867.37 & 49.88 \\
\hline min-alluip & $226(135,91)+5$ & 4890.67 & 45.2 \\
\hline alluip-active & $226(135,91)+5$ & $\mathbf{4 8 6 6 . 9 4}$ & 47.7 \\
\hline alluip-inclusive & $225(\mathbf{1 3 8}, 87)+4$ & 4958.49 & 52.12 \\
\hline alluip-exclusive & $223(134,89)+2$ & 5015.23 & $\mathbf{4 3 . 2}$ \\
\hline
\end{tabular}

Fig. 2. Results of MapleCOMSPS-LRB with 1-UIP, pure-alluip, min-alluip, alluip-active, alluip-inclusive, and alluip-exclusive on SAT2019 race main track.

Figure 2 shows each learning scheme's solved instances count, PAR-2 score, and average learnt clause size. We found that the stable-alluip learning schemes improved solved instances, PAR-2 scores, and learnt clause size over 1-UIP. More specifically, pure-alluip solved the most instances $(+7$ over 1 -UIP) and the most UNSAT instances $(+4)$; alluip-inclusive solved the most SAT instances $(+6)$; and alluip-active yields the best PAR-2 score (-151 than 1-UIP). In all cases the stable-alluip schemes learnt significantly smaller clauses on average.

Clause Reduction with stable-alluip. To precisely measure stable-alluip's clause reduction power, we compare each instance's learnt clause size from min-alluip and pure-alluip against 1-UIP. Figure 3 shows the probability density distribution (PDF) of the relative clause size of the stable-alluip learning schemes (min-alluip in green and pure-alluip in red) for each instance. min-alluip (pure-alluip resp.) produces shorter clauses for $88.5 \%(77.7 \%)$ of instances, and the average relative reduction ratio over 1 -UIP is $18.5 \%$ (9.6\%). Figure 4 compares the average learnt clause size of min-alluip, pure-alluip and 1-UIP per instance. Both stable-alluip schemes generally yield smaller clauses, and the size reduction is more significant for instances with larger 1-UIP clauses. 


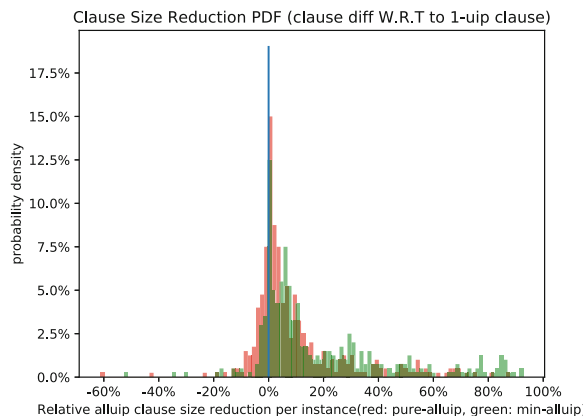

Fig. 3. Relative clause size reduction distribution. The $X$ axis indicates the relative size of difference between allUIP and 1-UIP clauses (calculated as $\left.\frac{\left|C_{1}\right|-\left|C_{i}\right|}{\left|C_{1}\right|}\right)$ for each instance, and the $Y$ axis shows the probability density. (Color figure online)

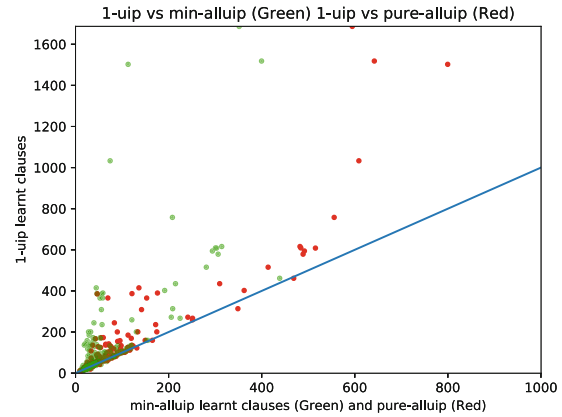

Fig. 4. Average clause size comparison plot. Each point in the plot represents an instance. The $X$ and $Y$ axes shows the clause length from stable-alluip and 1-UIP, respectively. Each green (red) dot represents an compared instance between MapleCOMSPS-LRB and Maple-minalluip (pure-alluip). (Color figure online)

The results in Fig. 2, 3 and 4 indicate min-alluip often achieves higher clause reduction than pure-alluip. We also observed that min-alluip attempted algorithm 1 more frequently than pure-alluip ( $28.8 \%$ vs $16.1 \%)$, and is more likely to succeed (59.3\% vs $43.4 \%)$. This observation agrees with our experiment results.

Reduced Proof Sizes with stable-alluip. A learning scheme that yields smaller clauses (lemmas) might also construct smaller causal proofs. For 88 UNSAT instances solved mutually by pure-alluip, min-alluip and 1-UIP schemes, we additionally compared the size of the optimized DRAT proof from the three learning schemes. We used the DRAT-trim tool [23] with a 30000 second timeout to check and optimize every DRAT proof once ${ }^{7}$.

The average optimized DRAT proof from min-alluip and pure-alluip are 556.6MB and 698.5MB, respectively. Both sizes are significantly smaller than the average optimized proof size from 1-UIP, $824.9 \mathrm{MB}$. The average proof size reduction per instance for min-alluip and pure-alluip is $16.5 \%$ and $3.6 \%$ against 1-UIP, which roughly correlate with our clause size observation in Fig. 3.

stable-alluip in Modern SAT Solvers. To validate stable-alluip in modern SAT solvers, we implemented stable-alluip in the winners of 2017, 2018 and 2019 SAT Race $[10,16,22]$ and in the expMaple-CM-GCBumpOnlyLRB [11] (expMaple) and CaDiCaL [4] solvers. expMaple is a top ten solver from 2019 SAT race which uses random walk simulation to help branching. We chose expMaple because the random walk simulation branching heuristic is different from local branching heuristic (VSIDS and LRB) that we have considered in

\footnotetext{
${ }^{7}$ Applying DRAT-trim multiple times can further reduce the proof size until a fixpoint. However, the full optimization is too time consuming for our experiments.
} 


\begin{tabular}{|l|l|l|l|}
\hline Solver & \#solved (SAT, UNSAT) & PAR-2 & avg. clause Size \\
\hline $\begin{array}{l}\text { SAT 2017 Winner } \\
\text { MapleLCMDist }\end{array}$ & $232(135,97)$ & 4755.96 & 61.9 \\
\hline MapleLCMDist-all-pure & $\mathbf{2 4 4}(\mathbf{1 4 6}, \mathbf{9 8})+12$ & $\mathbf{4 5 0 4 . 1 8}$ & 43.76 \\
\hline MapleLCMDist-all-min & $240(144,96)+8$ & 4601.25 & $\mathbf{3 6 . 9 7}$ \\
\hline MapleLCMDist-all-act & $237(140,97)+5$ & 4678.434 & 43.62 \\
\hline MapleLCMDist-all-inclusive & $234(137,97)+2$ & 4718.03 & 37.96 \\
\hline $\begin{array}{l}\text { SAT 2018 Winner } \\
\text { MapleCB }\end{array}$ & $236(138,98)$ & 4671.81 & 61.69 \\
\hline MapleCB-all-pure & $\mathbf{2 4 1}(\mathbf{1 4 2}, \mathbf{9 9})+5$ & $\mathbf{4 5 9 8 . 1 8}$ & 44.19 \\
\hline MapleCB-all-min & $236(141,95)+0$ & 4683.92 & 38.05 \\
\hline MapleCB-all-act & $240(141, \mathbf{9 9})+4$ & 4626.99 & 41.16 \\
\hline MapleCB-all-inclusive & $240(\mathbf{1 4 2}, 98)+4$ & 4602.13 & $\mathbf{3 7 . 5 2}$ \\
\hline
\end{tabular}

Fig. 5. Benchmark results of 1-UIP, pure-alluip. min-alluip, alluip-active and alluip-inclusive on SAT2019 race main track instances.

\begin{tabular}{|l|l|l|l|}
\hline $\begin{array}{l}\text { SAT } 2019 \text { Winner } \\
\text { Maple } C B-D L\end{array}$ & $238(140, \mathbf{9 8})$ & 4531.24 & 60.91 \\
\hline Maple $C B-D L$-all-pure & $240(142, \mathbf{9 8})+2$ & 4519.08 & 43.32 \\
\hline Maple $C B$ - $D L$-all-min & $244(\mathbf{1 4 8}, 96)+6$ & $\mathbf{4 4 1 9 . 8 4}$ & $\mathbf{3 6 . 8 8}$ \\
\hline MapleCB-DL-all-act & $243(146,97)+5$ & 4476.73 & 40.65 \\
\hline MapleCB-DL-all-inclusive & $\mathbf{2 4 3}(\mathbf{1 4 8}, 95)+5$ & 4455.76 & 37.02 \\
\hline $\begin{array}{l}\text { SAT } 2019 \text { Competitor } \\
\text { expMaple }\end{array}$ & $237(137,100)$ & 4628.96 & 63.19 \\
\hline expMaple-all-pure & $235(136,99)-2$ & 4668.96 & 48.26 \\
\hline expMaple-all-min & $241(143,98)+4$ & 4524.28 & 46.29 \\
\hline expMaple-all-act & $244(143, \mathbf{1 0 1})+7$ & $\mathbf{4 4 6 0 . 9 2}$ & 47.25 \\
\hline expMaple-all-inclusive & $\mathbf{2 4 5}(\mathbf{1 4 6}, 99)+8$ & 4475.76 & $\mathbf{4 5 . 3 3}$ \\
\hline $\begin{array}{l}\text { CaDiCaLversion } 1.2 .1 \\
\text { CaDiCaL-default }\end{array}$ & $249(150,99)$ & $\mathbf{4 3 1 1 . 7 6}$ & 101.96 \\
\hline CaDiCaL-default-all-pure & $248(151,97)-1$ & 4373.38 & 82.44 \\
\hline CaDiCaL-default-all-min & $248(149,99)-1$ & 4398.31 & 43.93 \\
\hline CaDiCaL-default-all-act & $\mathbf{2 5 2}(152, \mathbf{1 0 0})+3$ & 4331.56 & 47.34 \\
\hline CaDiCaL-default-all-inclusive & $251(\mathbf{1 5 3}, 98)+2$ & 4335.88 & $\mathbf{4 2 . 6 1}$ \\
\hline
\end{tabular}

Fig. 6. Benchmark results of 1-UIP, pure-alluip. min-alluip, alluip-active and alluip-inclusive on SAT2019 race main track instances.

alluip-active, alluip-inclusive, and alluip-exclusive. We chose CaDiCaL because its default configuration ( $C a D i C a L$-default) solved the most instances in the 2019 SAT Race (244). For this experiment, we used the latest available version of CaDiCaL-default instead of the 2019 SAT Race version [3]. We compared these solvers' base 1-UIP learning scheme with pure-alluip, min-alluip and the top two stable-alluip variants, alluip-active and alluip-inclusive, on the SAT Race 2019 main track benchmarks. We report solved instances, PAR-2 score and the average clause size. 
Figures 5 and 6 show the results of the stable-alluip configurations in our suite of modern solvers. Overall, we observed similar performance gain on all modern solvers as we have seen on MapleCOMSPS-LRB in Fig. 2. More specifically, almost all configurations improved on solved instance $(+3.9$ instances in average) and PAR-2 score ( -57.7 in average). The average clause size reduction is consistent across all solvers. Each configuration also exhibits different strengths: pure-alluip solved the most instances with the best PAR-2 score on two solvers, min-alluip yields small clauses, alluip-inclusive solved the most SAT instances, and alluip-active has stable performance.

On the SAT 2017 race winner MapleLCMDist, all four configurations of stable-alluip solved more instances than 1-UIP learning. pure-alluip solved more UNSAT and SAT instances while the other configurations improved on solving SAT instances. The clause size reduction of stable-alluip is more significant on this solver than on MapleCOMSPS-LRB. The SAT 2018 race winner Maple $C B$ uses chronological backtracking $(\mathrm{CB})$; three out of four configurations outperformed 1-UIP. On the SAT 2019 race winner MapleCB-DL, all four stable-alluip configurations solved more instances than 1-UIP. MapleCB-DL prioritizes clauses that are learned multiple times. We observed that stable-alluip clauses are less likely to be duplicated. As an example, min-alluip on average, added $12 \%$ less duplicated clauses into the core clause database than 1-UIP. This observation is surprising, and the cause is unclear.

On expMaple, three out of four stable-alluip configurations solved more instances than 1-UIP learning. We noticed that both alluip-active and alluip-inclusive show better performance than min-alluip and pure-alluip on this solver. The random walk simulation branching heuristic, however, didn't impact the performance of stable-alluip schemes significantly.

CaDiCaL-default with 1-UIP solved 249 instances. Applying alluip-active and alluip-inclusive helped the solver solve 3 and 2 more instances, respectively. The 1-UIP clauses in $\mathrm{CaDiCaL}$-default were much larger than other solvers on average (101 vs 60) but the stable-alluip configurations yielded similar clause sizes.

\section{Conclusion}

In this paper we introduced a new clause learning scheme, stable-alluip, that preserves the strengths 1-UIP learning while learning shorter clauses. We provided empirical evidence that using stable-alluip and its variants in modern CDCL solvers achieves significant clause reduction and yields useful performance gains.

Our scheme extends 1-UIP learning by performing further resolution beyond the deepest decision level in an attempt to find the UIP at each level in the learnt clause. Since resolutions may increase the clause's LBD by introducing literals from new decision levels, we presented two methods to block such literals from entering the clause. Although our learning scheme is conceptually simple, and we presented optimizations to reduce and balance the learning cost. We additionally presented variants of our schemes to account for features used in state of the art solvers, e.g., local branching heuristics and chronological backtracking. 
Although the field of SAT solving has converged on using the 1-UIP learning scheme, we have shown the possibility of developing an effective alternative through understanding the strengths and weaknesses of 1-UIP and clause learning schemes. Our learning scheme can be generalized and further improved by exploring more fine-grained trade-offs between different clause quality metrics beyond clause size and LBD. We also plan to study the interaction between clause learning and variable branching. Since most of the branching heuristics are tailored for 1-UIP scheme, their interactions with other learning schemes requires further study.

\section{References}

1. Audemard, G., Simon, L.: Predicting learnt clauses quality in modern SAT solvers. In: Boutilier, C. (ed.) IJCAI 2009, Proceedings of the 21st International Joint Conference on Artificial Intelligence, Pasadena, California, USA, 11-17 July 2009, pp. 399-404 (2009). http://ijcai.org/Proceedings/09/Papers/074.pdf

2. Beame, P., Kautz, H.A., Sabharwal, A.: Towards understanding and harnessing the potential of clause learning. J. Artif. Intell. Res. 22, 319-351 (2004). https:// doi.org/10.1613/jair.1410

3. Biere, A.: CADICAL at the SAT race 2019. In: Heule, M.J.H., Järvisalo, M., Suda, M. (eds.) Proceedings of SAT Competition 2019 Solver and Benchmark Descriptions. University of Helsinki (2019). http://hdl.handle.net/10138/306988

4. Biere, A.: Cadical SAT solver (2019). https://github.com/arminbiere/cadical

5. Bayardo Jr, R.J., Schrag, R.: Using CSP look-back techniques to solve real-world SAT instances. In: Kuipers, B., Webber, B.L. (eds.) Proceedings of the Fourteenth National Conference on Artificial Intelligence and Ninth Innovative Applications of Artificial Intelligence Conference, AAAI 97, IAAI 97, 27-31 July 1997, Providence, Rhode Island, USA, pp. 203-208. AAAI Press/The MIT Press (1997). http://www. aaai.org/Library/AAAI/1997/aaai97-032.php

6. Katebi, H., Sakallah, K.A., Marques-Silva, J.P.: Empirical study of the anatomy of modern SAT solvers. In: Sakallah, K.A., Simon, L. (eds.) SAT 2011. LNCS, vol. 6695, pp. 343-356. Springer, Heidelberg (2011). https://doi.org/10.1007/9783-642-21581-0_27

7. Knuth, D.E.: Implementation of algorithm 7.2.2.2c (conflict-driven clause learning SAT solver). https://www-cs-faculty.stanford.edu/ knuth/programs/sat13.w

8. Liang, J.H., Ganesh, V., Poupart, P., Czarnecki, K.: Learning rate based branching heuristic for SAT solvers. In: Creignou, N., Le Berre, D. (eds.) SAT 2016. LNCS, vol. 9710, pp. 123-140. Springer, Cham (2016). https://doi.org/10.1007/978-3-31940970-2_9

9. Liang, J.H., Oh, C., Ganesh, V., Czarnecki, K., Poupart, P.: Maple-COMSPS, MapleCOMSPS_LRB, MapleCOMSPS_CHB. In: Balyo, T., Heule, M.J.H., Järvisalo, M.J. (eds.) Proceedings of SAT Competition 2016 Solver and Benchmark Descriptions. University of Helsinki (2016). http://hdl.handle.net/10138/164630

10. Luo, M., Li, C., Xiao, F., Manyà, F., Lü, Z.: An effective learnt clause minimization approach for CDCL SAT solvers. In: Sierra, C. (ed.) Proceedings of the Twenty-Sixth International Joint Conference on Artificial Intelligence, IJCAI 2017, Melbourne, Australia, 19-25 August 2017, pp. 703-711 (2017). ijcai.org, https:// doi.org/10.24963/ijcai.2017/98 
11. Chowdhury, M.S., Müller, M., You, J.H.: Four CDCL SAT solvers based on exploration and glue variable bumping. In: Heule, M.J.H., Järvisalo, M., Suda, M. (eds.) Proceedings of SAT Competition 2019 Solver and Benchmark Descriptions. University of Helsinki (2019). http://hdl.handle.net/10138/306988

12. Möhle, S., Biere, A.: Backing backtracking. In: Janota, M., Lynce, I. (eds.) SAT 2019. LNCS, vol. 11628, pp. 250-266. Springer, Cham (2019). https://doi.org/10. 1007/978-3-030-24258-9_18

13. Moskewicz, M.W., Madigan, C.F., Zhao, Y., Zhang, L., Malik, S.: Chaff: engineering an efficient SAT solver. In: Proceedings of the 38th Design Automation Conference, DAC 2001, Las Vegas, NV, USA, 18-22 June 2001, pp. 530-535. ACM (2001). https://doi.org/10.1145/378239.379017

14. Nadel, A., Ryvchin, V.: Chronological backtracking. In: Beyersdorff, O., Wintersteiger, C.M. (eds.) SAT 2018. LNCS, vol. 10929, pp. 111-121. Springer, Cham (2018). https://doi.org/10.1007/978-3-319-94144-8_7

15. Pipatsrisawat, K., Darwiche, A.: On the power of clause-learning SAT solvers as resolution engines. Artif. Intell. 175(2), 512-525 (2011). https://doi.org/10.1016/ j.artint.2010.10.002

16. Ryvchin, V., Nadel, A.: Maple_LCM_Dist ChronoBT: featuring chronological backtracking. In: Heule, M.J.H., Järvisalo, M., Suda, M. (eds.) Proceedings of SAT Competition 2018 Solver and Benchmark Descriptions. University of Helsinki (2018). https://hdl.handle.net/10138/237063

17. Sabharwal, A., Samulowitz, H., Sellmann, M.: Learning back-clauses in SAT. In: Cimatti, A., Sebastiani, R. (eds.) SAT 2012. LNCS, vol. 7317, pp. 498-499. Springer, Heidelberg (2012). https://doi.org/10.1007/978-3-642-31612-8_53

18. Silva, J.P.M., Lynce, I., Malik, S.: Conflict-driven clause learning SAT solvers. In: Handbook of Satisfiability, pp. 131-153. IOS Press (2009). https://doi.org/10. 3233/978-1-58603-929-5-131

19. Silva, J.P.M., Sakallah, K.A.: GRASP - a new search algorithm for satisfiability. In: Rutenbar, R.A., Otten, R.H.J.M. (eds.) Proceedings of the 1996 IEEE/ACM International Conference on Computer-Aided Design, ICCAD 1996, San Jose, CA, USA, 10-14 November 1996, pp. 220-227. IEEE Computer Society/ACM (1996). https://doi.org/10.1109/ICCAD.1996.569607

20. Silva, J.P.M., Sakallah, K.A.: GRASP: a search algorithm for propositional satisfiability. IEEE Trans. Comput. 48(5), 506-521 (1999). https://doi.org/10.1109/12. 769433

21. Sörensson, N., Biere, A.: Minimizing learned clauses. In: Kullmann, O. (ed.) SAT 2009. LNCS, vol. 5584, pp. 237-243. Springer, Heidelberg (2009). https://doi.org/ 10.1007/978-3-642-02777-2_23

22. Kochemazov, S., Zaikin, O., Kondratiev, V., Semenov, A.: MapleLCMD istchronoBT-DL, duplicate learnts heuristic-aided solvers at the SAT race 2019. In: Heule, M.J.H., Järvisalo, M., Suda, M. (eds.) Proceedings of SAT Competition 2019 Solver and Benchmark Descriptions. University of Helsinki (2019). http:// hdl.handle.net/10138/306988 
23. Wetzler, N., Heule, M.J.H., Hunt, W.A.: DRAT-trim: efficient checking and trimming using expressive clausal proofs. In: Sinz, C., Egly, U. (eds.) SAT 2014. LNCS, vol. 8561, pp. 422-429. Springer, Cham (2014). https://doi.org/10.1007/978-3-31909284-3_31

24. Wieringa, S., Heljanko, K.: Concurrent clause strengthening. In: Järvisalo, M., Van Gelder, A. (eds.) SAT 2013. LNCS, vol. 7962, pp. 116-132. Springer, Heidelberg (2013). https://doi.org/10.1007/978-3-642-39071-5_10

25. Zhang, L., Madigan, C.F., Moskewicz, M.W., Malik, S.: Efficient conflict driven learning in Boolean satisfiability solver. In: Ernst, R. (ed.) Proceedings of the 2001 IEEE/ACM International Conference on Computer-Aided Design, ICCAD 2001, San Jose, CA, USA, 4-8 November 2001, pp. 279-285. IEEE Computer Society (2001). https://doi.org/10.1109/ICCAD.2001.968634 